\title{
Performance Prediction of Refractured Wells Based on Embedded Discrete-Fracture Modeling Method
}

\author{
Yawen Du $(\mathbb{D}$, Xiaolu Cao $\mathbb{D}$, and Bailu Teng $\mathbb{C}$ \\ School of Energy Resources, China University of Geosciences (Beijing), Beijing 100083, China \\ Correspondence should be addressed to Bailu Teng; bailu@cugb.edu.cn
}

Received 28 October 2021; Revised 27 November 2021; Accepted 2 December 2021; Published 17 January 2022

Academic Editor: Wei Yu

Copyright (C) 2022 Yawen Du et al. This is an open access article distributed under the Creative Commons Attribution License, which permits unrestricted use, distribution, and reproduction in any medium, provided the original work is properly cited.

\begin{abstract}
In unconventional reservoirs, the production rate of the initially fractured well will decrease rapidly as the development proceeds. In order to maintain economic well productivity of the initial fracture, the industries can create refractures to prevent the well production rate from decreasing. However, the production of the initial fracture can induce the stress field to reorientate, which will cause the refracture to propagate along a different direction from the initial fracture. Thus, the refracturing treatment can result in a complex fracture system which raises a stringent challenge for one to evaluate the performance of the refractured well. In this paper, the authors utilize the embedded discrete fracture model (EDFM) to characterize the performance of the refractured wells. The calculated results in this paper show that, for the same equivalent matrix permeability, a larger permeability component parallel to the initial fracture can lead to a higher increase ratio of the refracturing treatment but lower cumulative production. If the reoriented section of the refracture is orthogonal to the initial fracture, the productivity of the refracture system can be the highest. The length of the reoriented section plays a more important role than the length of the initially oriented section in influencing the refracturing treatment. The refracturing treatment can lead to a higher increase of cumulative production if the initial fracture has a lower permeability.
\end{abstract}

\section{Introduction}

In recent years, the refracturing treatment has increasingly attracted the attention of industries to improve the productivity of initially fractured well. Hydraulic fractures will orient along the direction that is parallel to the maximum principal stress (or perpendicular to the least principal stress) $[1,2]$. Since the production of the initial fracture can result in a stress reorientation around the fractured well $[3,4]$, the refracturing treatment will induce a fracture that has a different growth direction from the initial fracture. Figure 1 shows the schematic of the refracture system. As shown in Figure 1, because of the effect of stress reorientation, the refracture will first penetrate the reservoir along a direction that has a certain azimuth with respect to the initial fracture. As the fracture grows beyond the stress reorientation region, the propagation direction of the refracture will gradually change to the direction of the initial fracture. Thus, the refracture can be divided into three sections, including a reorientated section (red line in Figure 1), a transition section (yellow line in Figure 1), and an initially orientated section (blue line in Figure 1). Shan et al. provided a pressure transient analysis model to posterior assess the fracture propagation of refractured vertical well in oil reservoir [5]. Yi and Sharma proposed a new method to calculate slurry distribution among multiple fractures during the fracturing treatment and refracturing treatment [6]. Wang and Salehi used hybrid simulation with neural network and data analysis techniques to select refracture candidates [7]. Li et al. examine the stress redistribution and fracture propagation during restimulation of shale gas reservoirs [8].

For the complex fracture system that is shown in Figure 1, the conventional numerical method can be used to predict the production of the refractured well. In the conventional numerical method, the local refined grid (LGR) or unstructured grid will be applied to characterize the trajectory of the refracture. However, both the LGR technique and the unstructured grid technique will increase the 


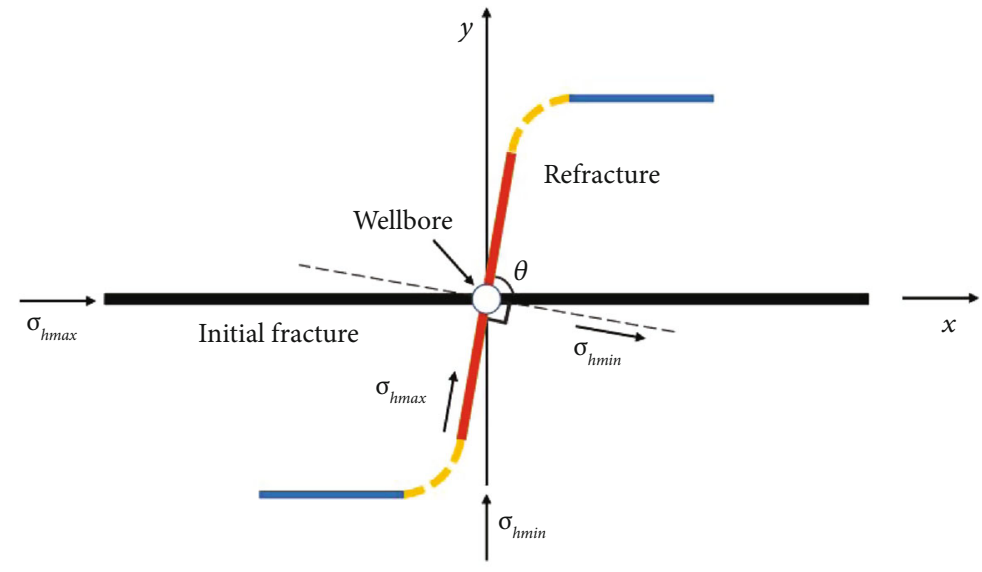

FIGURE 1: Schematic of the refractured system.

workload and decrease the computational efficiency of the conventional numerical simulation method, which makes these two techniques unattractive for simulating the refractured well. Compared to the conventional numerical method, the semianalytical method provides a more computationally efficient way to simulate the performance of various complex fracture systems. Since only the fracture system needs to be discretized in the semianalytical method, the simulation efficiency can be significantly improved. Wang et al. studied the transient flow of fractured media with fractal geometry [9]. Luo et al. proposed a new fracture-unit model on the basis of the semi-analytical method and applied the proposed model to a Z-fold fracture [10]. Liu et al. developed a semianalytical model to simulate the scenario of multiples vertical well considering the effect of well interference [11]. Xu et al. predicted the production of multiwing fractured well with finite conductivity in naturally fractured reservoirs with the aid of a semianalytical model [12]. Zhang and Emami-Meybodi used the semianalytical method to conduct rate transient analysis in shale gas reservoirs by accounting for the two-phase flowback [13]. Although the semianalytical method provides an alternate method for simulating complex fractures, it can be difficult to characterize the reservoir heterogeneity and irregular reservoir boundary. Hence, for field applications, the semianalytical method is commonly used to conduct postfracture analysis with early production data rather than to predict the long-term well performance.

In recent years, the embed discrete fracture model (EDFM) has attracted more and more attention for modeling complex fractures. The EDFM was developed by Lee et al. to honor the complex fracture geometry while using structured grids [14]. Sangnimnuan et al. proposed a fluidflow/geomechanic model to efficiently predict the stress evolution in unconventional reservoirs with complex fractures based on EDFM [15]. Xu and Sepehrnoori built a fieldscale reservoir simulator with the EDFM by use of cornerpoint grids [16]. Yao et al. combined the EDFM with a dual-porosity/dual-permeability model to parameterize multiscale fractures [17].
According to the aforementioned argument, one can conclude that first, the conventional numerical method is not convenient to handle the problems of the complex fractures; second, although the semianalytical method can be used to characterize the complex fractures, it can be challenging to consider reservoir heterogeneity and irregular boundaries; and third, EDFM is an extension to the conventional numerical method and can be utilized to simulate various complex fractures. This study, therefore, is designed to carry out a thorough study of the performance of the refractured well based on EDFM.

\section{Methodology}

The EDFM characterizes the matrix-fracture flow and fracture-fracture flow through nonneighboring connection (NNC) formulations [14]. There are three types of NNC in EDFM, including type \#1, the connection between fracture segment and matrix grid, type \#2, the connection between fracture segments of the same fracture, and type \#3, the connection between fracture segments of intersected fractures. Figure 2 shows all three types of NNCs. In this paper, since there is no fracture intersection, only NNC type \#1 and type $\# 2$ are considered.

For the sake of convenience, the fluid flow between two continuous mediums is generally described as

$$
q_{a-b}=T_{a-b}\left(p_{a}-p_{b}\right)
$$

where $q$ is the flux rate $\left(\mathrm{m}^{3} /\right.$ day), $T$ is the transmissibility $\left(\mathrm{m}^{3} /(\right.$ day $\left.\bullet \mathrm{MPa})\right), p$ is the pressure $(\mathrm{MPa})$, and subscripts $a$ and $b$ indicate the two continuous mediums. For example, the fluid flow between matrix grid and fracture segment can be expressed as

$$
q_{m-f}=T_{m-f}\left(p_{m}-p_{f}\right)
$$

Thus, only the calculation method of the transmissibility needs to be changed to describe the fluid flow between 


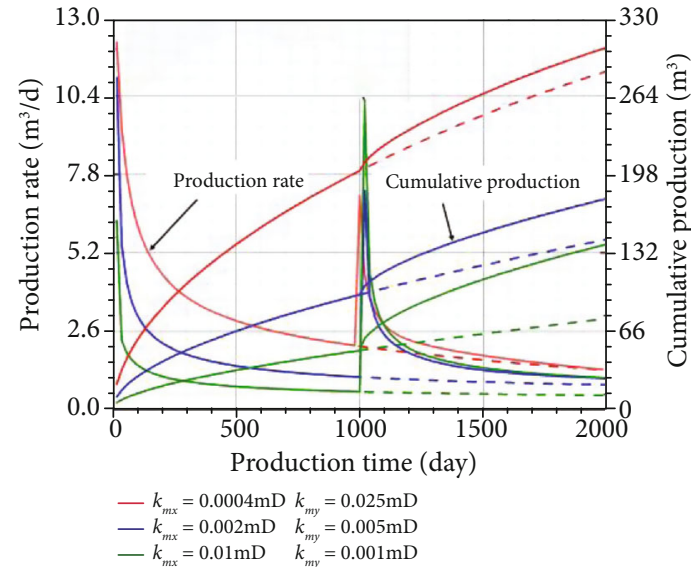

(a)

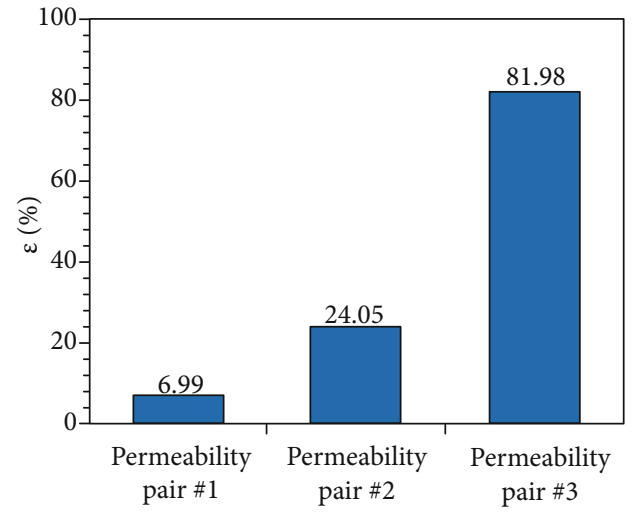

(b)

FIGURE 3: Performance of the refracturing treatment with different permeability anisotropies: (a) production rate and cumulative production and (b) comparison of the increase ratio.

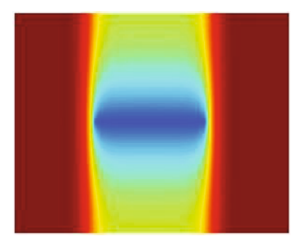

(a)

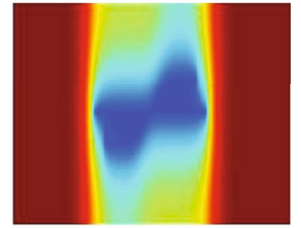

(d)

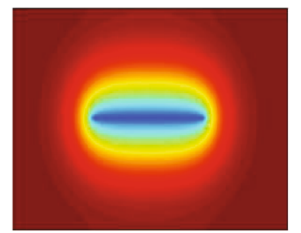

(b)

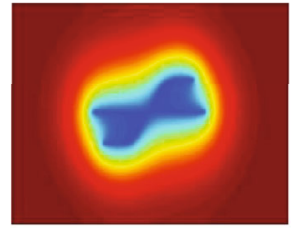

(e)

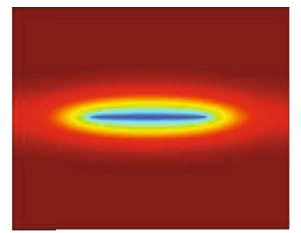

(c)

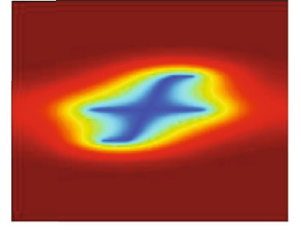

(f)

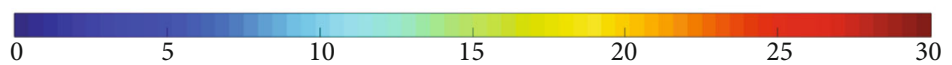

FIGURE 4: Pressure fields of the $2000^{\text {th }}$ day $(\mathrm{MPa})$ : (a) permeability pair \#1 without refracturing treatment, (b) permeability pair \#2 without refracturing treatment, (c) permeability pair \#3 without refracturing treatment, (d) permeability pair \#1 with refracturing treatment, (e) permeability pair \#2 with refracturing treatment, and (f) permeability pair \#3 with refracturing treatment.

one can observe Forchheimer flow. For such scenarios, one can use the method introduced in Al-Rbeawi [22] to consider the high rate non-Darcy flow.

\section{Results and Discussion}

In this part, a comprehensive investigation was conducted to study the effects of permeability anisotropy, refracture azimuth, fracture length, and fracture permeability on the performance of the refractured well. The benchmark values of the parameters used in this section are as follows: initial reservoir pressure $p_{i}=30 \mathrm{MPa}$, reservoir dimension along $x$ -axis $X_{e}=500 \mathrm{~m}$, reservoir dimension along $y$-axis $Y_{e}=400$ $\mathrm{m}$, reservoir thickness $h=15 \mathrm{~m}$, reservoir permeability along $x$-axis $k_{m x}=0.01 \mathrm{mD}$, reservoir permeability along $y$-axis $k_{m y}=0.01 \mathrm{mD}$, matrix porosity $\phi_{m}=0.2$, initial fracture porosity $\phi_{f 1}=0.2$, refracture porosity $\phi_{f 2}=0.2$, total com- pressibility of the matrix system $c_{t m}=0.0012 \mathrm{MPa}^{-1}$, total compressibility of initial fracture $c_{t f 1}=0.0012 \mathrm{MPa}^{-1}$, total compressibility of refracture $c_{t f 2}=0.0012 \mathrm{MPa}^{-1}$, initial fracture length $L_{f 1}=100 \mathrm{~m}$, length of the reoriented section of the refracture $L_{f 2 r}=50 \mathrm{~m}$, length of the initially orientated section $L_{f 2 i}=30 \mathrm{~m}$, oil viscosity $\mu=1 \mathrm{mPa} \cdot \mathrm{s}$, initial fracture permeability $k_{f 1}=1 \times 10^{6} \mathrm{mD}$, refracture permeability $k_{f 2}$ $=1 \times 10^{6} \mathrm{mD}$, initial fracture width $w_{f 1}=1 \times 10^{-3} \mathrm{~m}$, refracture width $w_{f 2}=1 \times 10^{-3} \mathrm{~m}$, formation volume factor $B=$ 0.985 , wellbore pressure $p_{w}=5 \mathrm{MPa}$, production time only with the initial fracture $T_{f 1}=1000$ days, production time with both the initial fracture and refracture $T_{f 2}=1000$ days, and the azimuth between the refracture and initial fracture $\theta=90^{\circ}$. It is worth noting that the EDFM method is a numerical method for calculating the fluid flow in fractured reservoirs. Therefore, although the investigation was 


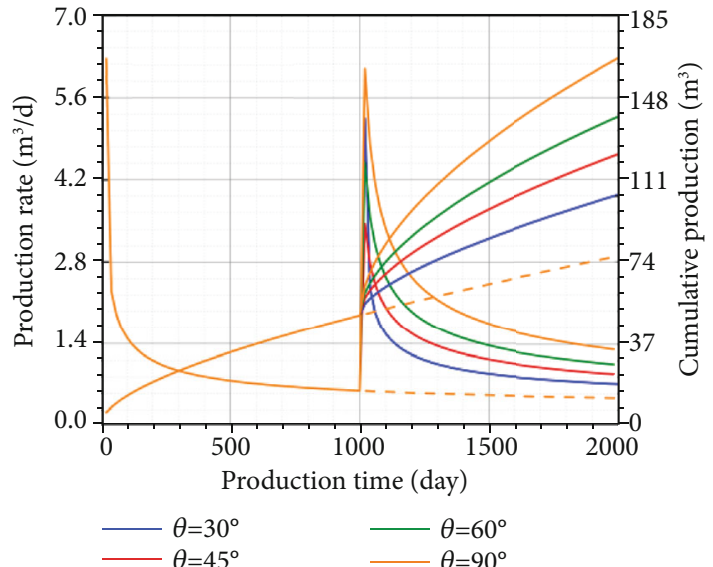

(a)

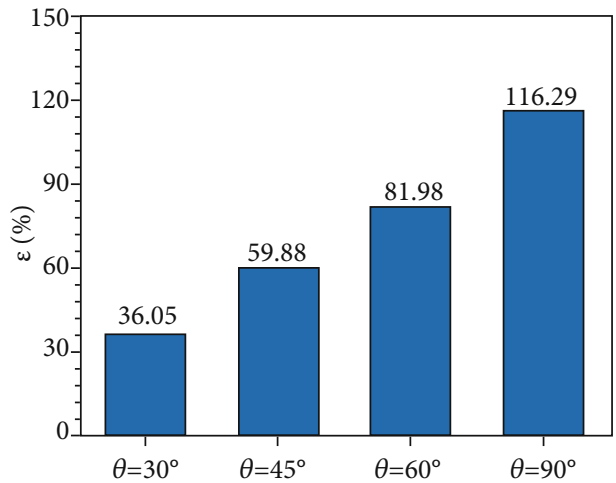

(b)

FIGURE 5: Performance of the refracturing treatment with different refracture azimuths: (a) production rate and cumulative production and (b) comparison of the increase ratio.

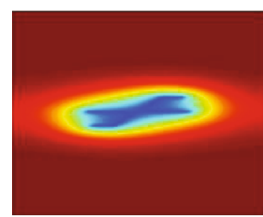

(a)

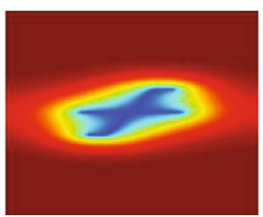

(b)

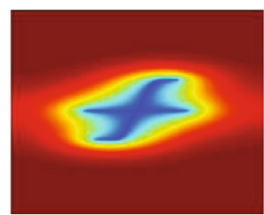

(c)

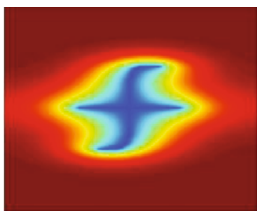

(d)

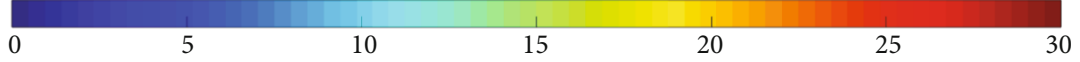

Figure 6: Pressure fields of the $2000^{\text {th }}$ day with different refracture azimuths (MPa): (a) $\theta=90^{\circ}$, (b) $\theta=45^{\circ}$, (c) $\theta=60^{\circ}$, and (d) $\theta=90^{\circ}$.

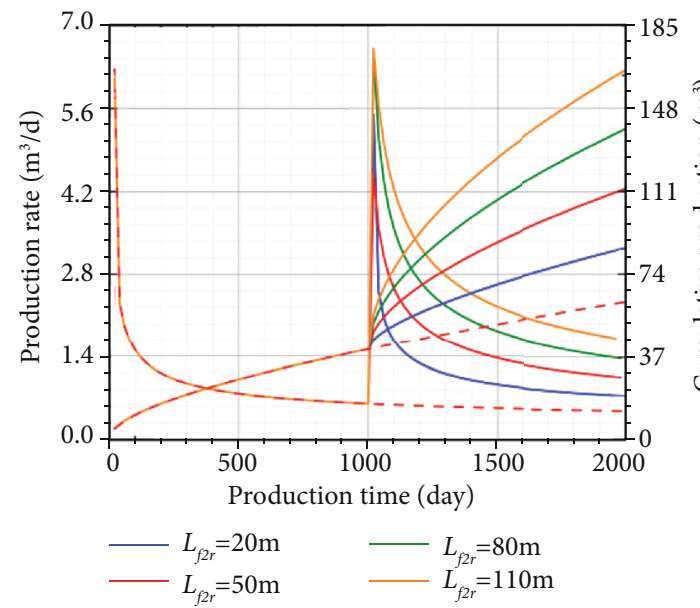

(a)

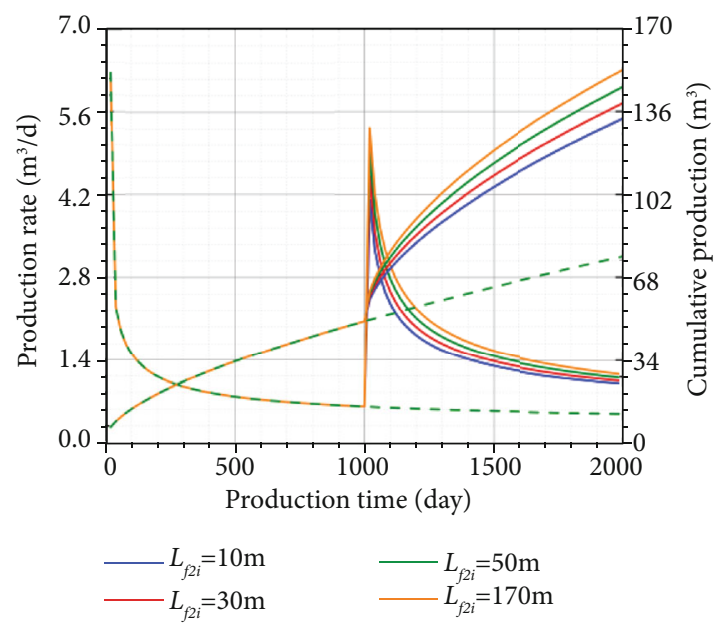

(b)

Figure 7: Performance of the refractured well with (a) different lengths of reoriented section and (b) different lengths of initially reoriented section.

conducted on a simplified model and the real reservoir geology is neglected, the introduced EDFM method can be applied in various sedimentary reservoirs if the model is sufficiently accurate to characterize the reservoir geology.
3.1. Permeability Anisotropy. Three pairs of permeability are examined to explore the effect of the permeability anisotropy on the performance of refractured well, including pair \#1 $k_{m x}=0.0004 \mathrm{mD}$ and $k_{m y}=0.025 \mathrm{mD}$, pair \#2 $k_{m x}=0.002$ $\mathrm{mD}$ and $k_{m y}=0.005 \mathrm{mD}$, and pair $\# 3 k_{m x}=0.01 \mathrm{mD}$ and 


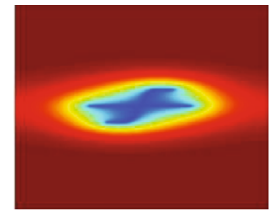

(a)

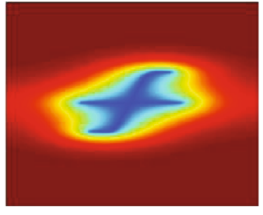

(b)

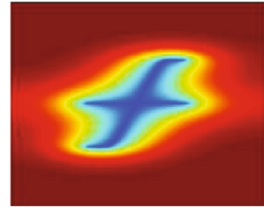

(c)

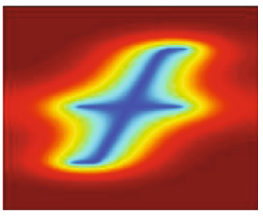

(d)

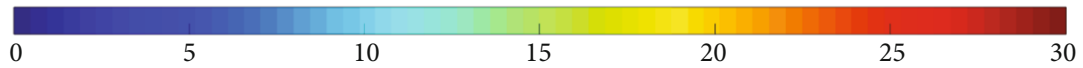

FIGURE 8: Pressure fields of the $2000^{\text {th }}$ day with different lengths of reoriented section (MPa): (a) $L_{f 2 r}=20 \mathrm{~m},(\mathrm{~b}) L_{f 2 r}=50 \mathrm{~m},(\mathrm{c}) L_{f 2 r}=80 \mathrm{~m}$, and (d) $L_{f 2 r}=110 \mathrm{~m}$.

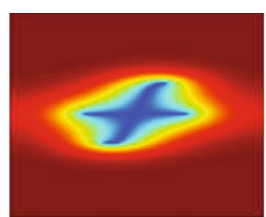

(a)

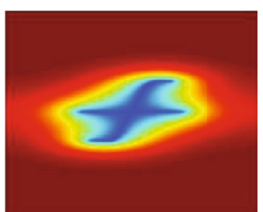

(b)

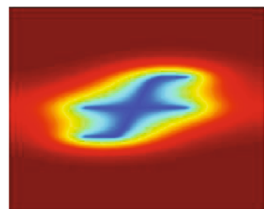

(c)

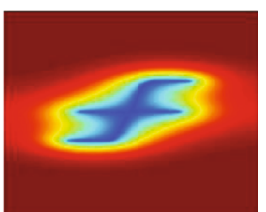

(d)

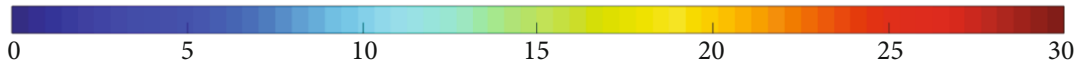

FIgure 9: Pressure fields of the $2000^{\text {th }}$ day with different lengths of initially oriented section (MPa): (a) $L_{f 2 i}=10 \mathrm{~m},(\mathrm{~b}) L_{f 2 i}=30 \mathrm{~m},(\mathrm{c})$ $L_{f 2 i}=50 \mathrm{~m}$, and (d) $L_{f 2 i}=70 \mathrm{~m}$.

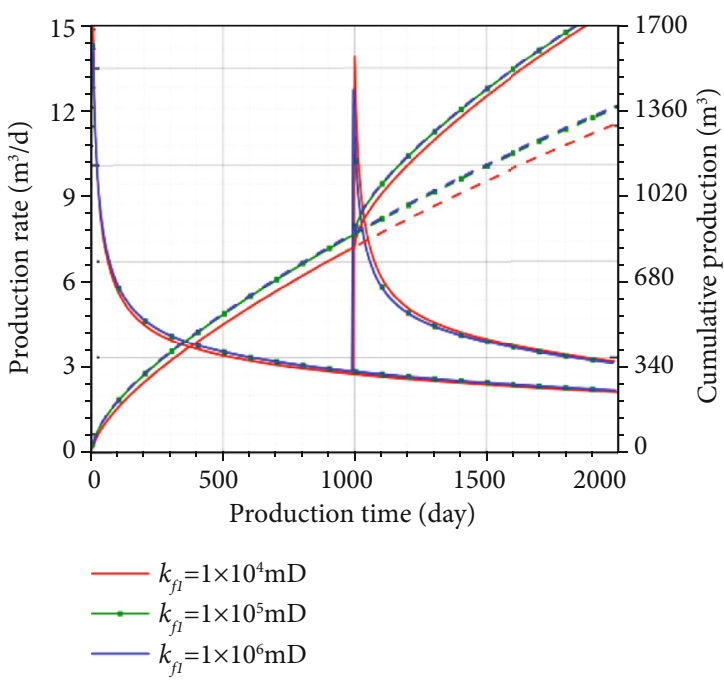

(a)

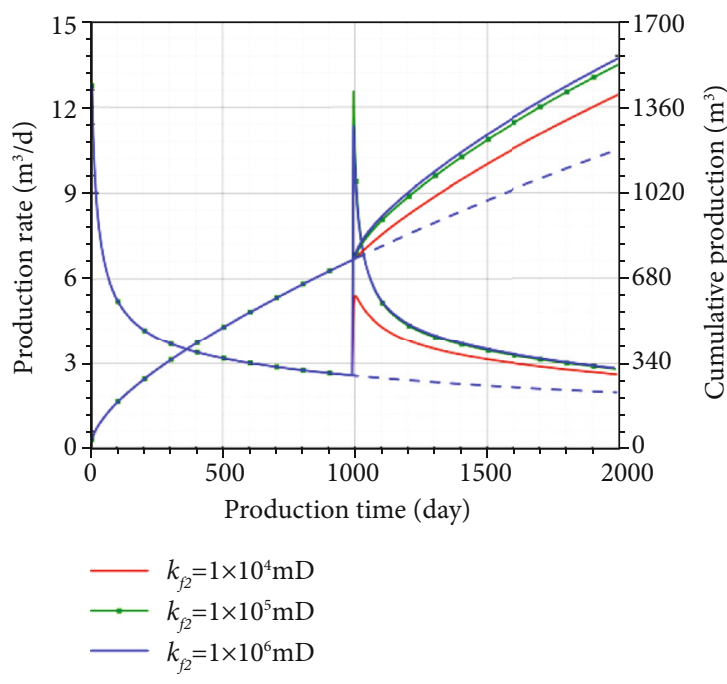

(b)

Figure 10: Performance of the refractured well with (a) different initial fracture permeability (b) different refracture permeability.

$k_{m y}=0.001 \mathrm{mD}$. As the permeability pair is varied from $\# 1$ to \#3, the permeability along the $x$-axis is increased while the permeability along the $y$-axis is decreased. It is worth noting that the value of $k_{m x} \times k_{m y}$ remains unchanged for these three anisotropic permeability pairs, such that the permeability of the equivalent isotropic permeability system will have the same value. Figure 3 compares the well performance of the refractured well with different permeability pairs. In Figure 3(a), the solid lines represent the well production with refracturing treatment, and the dash lines represent the well production without refracturing treatment.
As shown in Figure 3(a), as the permeability along $y$-axis is increased, the cumulative well production is increased, and the highest cumulative well production happens to the permeability pair \#1 with the largest permeability along $y$-axis. The increase ratio of the cumulative production $\varepsilon$ was further defined to compare the effect of the refracturing treatment:

$$
\varepsilon=\frac{Q_{\mathrm{re}}-Q_{\text {non }}}{Q_{\text {non }}} \times 100 \% \text {, }
$$




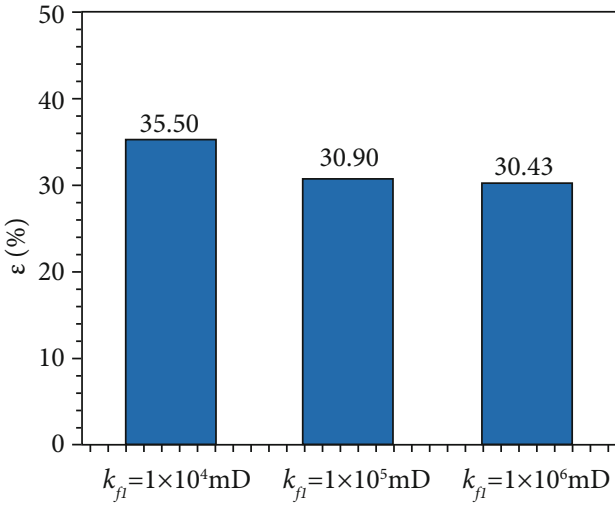

(a)

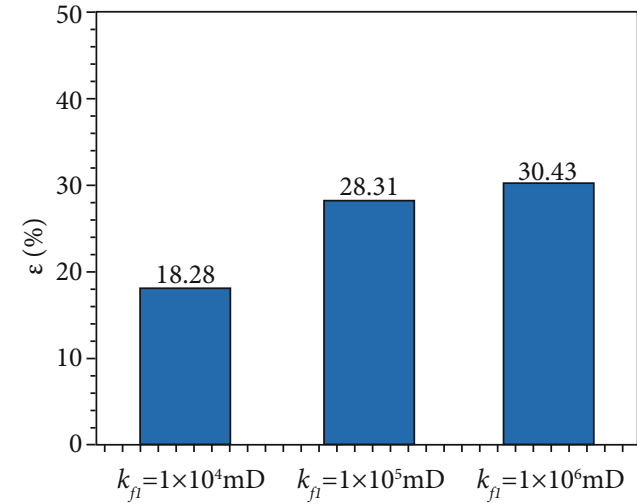

(b)

FIGURE 11: Increase ratio of the cumulative production with (a) different initial fracture permeability. (b) Different refracture permeability.

where $Q_{\text {re }}$ represents the cumulative production with refracturing treatment $\left(\mathrm{m}^{3}\right)$, and $Q_{\text {non }}$ represents the cumulative production without refracturing treatment $\left(\mathrm{m}^{3}\right)$. Figure 3(b) compares the increase ratio of different permeability anisotropies. It is shown in Figure 3(b) that permeability pair \#3 leads to the highest increase ratio of $81.98 \%$. According to the results demonstrated in Figure 3, one can find that a larger permeability perpendicular to the initial fracture can result in a larger cumulative production but lower efficiency of the refracturing treatment.

Figure 4 shows the pressure fields of the $2000^{\text {th }}$ day without refracturing treatment (Figures $4(\mathrm{a})-4(\mathrm{c})$ ) and with refracturing treatment (Figures $4(\mathrm{~d})-4(\mathrm{f})$ ) of different permeability anisotropies. Comparing Figure 4(a) to Figure 4(d), one can find that, for permeability pair \#1 which has the lowest $k_{m x}$ and highest $k_{m y}$, the refracturing treatment will only slightly increase the low-pressure area (the low-pressure area can be regarded as the area within which the reservoir pressure is much lower than the initial pressure. For example, in Figure 4, the green and blue areas in Figure 4 can be regarded as low-pressure areas). Thus, the cumulative production of permeability pair \#1 is least increased by the refracturing treatment. Figures $4(\mathrm{c})$ and 4(f) show the pressure field without refracturing treatment and with refracturing treatment, respectively, for permeability pair \#3. This suggests that the refracturing treatment can significantly increase the low-pressure area, leading to a distinguishable performance of the refracturing treatment.

3.2. Refracture Azimuth. The refracture azimuth is varied from $30^{\circ}$ to $90^{\circ}$ to explore the effect of the refracture azimuth on the performance of refractured well. Figure 5 demonstrates the performance of the refracturing treatment with various azimuths. It can be seen in Figure 5(a) that as the azimuth is ranged from $30^{\circ}$ to $90^{\circ}$, the cumulative production of the refracturing treatment is increased, indicating that making a refracture orthogonal to the initial fracture is favorable for increasing the well productivity. Figure 5(b) compares the increase ratio of the cumulative production, which also illustrates that a refracture that is orthogonal to the initial refracture $\left(\theta=90^{\circ}\right)$ induces the maximum increase of cumulative production. Figure 6 presents the pressure fields of the $2000^{\text {th }}$ day with different refracturing azimuths. It is observed in this figure that as the azimuth is increased, the low-pressure area is increased. Thus, the refracture azimuth of $\theta=90^{\circ}$ results in the highest cumulative production.

3.3. Refracture Length. Figures $7(a)$ and $7(b)$ show the performance of the refractured well with different lengths of reoriented section and different lengths of initially reoriented section, respectively. In Figure 7(a), the length of the reoriented section of $20 \mathrm{~m}, 50 \mathrm{~m}, 80 \mathrm{~m}$, and $110 \mathrm{~m}$ is considered, and in Figure 7(b), length of the initially oriented section of $10 \mathrm{~m}, 30 \mathrm{~m}, 50 \mathrm{~m}$, and $70 \mathrm{~m}$ is considered. It is observed in Figure 7 that a longer reoriented section and a longer initially oriented section can both induce a higher cumulative production. However, the increase of the reoriented section can lead to a more significant increase of the cumulative production, implying that the length of the reoriented section plays a more important role in affecting the refracturing treatment.

Figures 8 and 9 exhibit pressure fields of the $2000^{\text {th }}$ day with different lengths of reoriented section and different lengths of initially reoriented section, respectively. Comparing the pressure fields shown in Figure 8 to those shown in Figure 9, one can see that increasing the length of the reoriented section can render the low-pressure area expand to a larger area than increasing the length of the initially oriented section. Therefore, the reoriented section can exert a more obvious impact on the well performance than the initially oriented section.

3.4. Fracture Permeability. Figure 10(a) illustrates the performance of the refractured well with different initial fracture permeability $\left(k_{f 1}=1 \times 10^{4} \mathrm{mD}, k_{f 1}=1 \times 10^{5} \mathrm{mD}\right.$, and $k_{f 1}$ $=1 \times 10^{6} \mathrm{mD}$ ), whereas Figure $10(\mathrm{~b})$ illustrates the performance of the refractured well with different refracture permeability $\quad\left(k_{f 2}=1 \times 10^{4} \mathrm{mD}, \quad k_{f 2}=1 \times 10^{5} \mathrm{mD}, \quad\right.$ and $k_{f 2}=1 \times 10^{6} \mathrm{mD}$ ). As shown in Figure 10(a), larger initial fracture permeability and refracture permeability can both result in a larger cumulative well production. Figure $11 \mathrm{com}$ pares the increase ratio of the cumulative production with different initial fracture permeability (Figure 11(a)) and 
different refracture permeability (Figure 11(b)). An interesting observation is that as the initial fracture permeability is increased, the increase ratio is decreased, whereas as the refracture permeability is increased the increase ratio is increased. This demonstrates that the refracturing treating has more advantages to enhance the productivity of a lower permeability initial fracture, and the larger the permeability of the refracture, the higher performance of the refracturing treatment.

\section{Conclusions}

In this paper, the authors conducted a comprehensive study of the well performance of the refractured well by use of EDFM. The permeability anisotropy is also considered in this paper. The influencing factors, including permeability anisotropy, refracture azimuth, fracture length, and fracture permeability, are investigated. On the basis of the calculated results in this paper, the following conclusions can be formed:

(1) The performance of the refracturing treatment is highly dependent on the low-pressure area that is induced by the refracture. A better performance of the refracturing treatment commonly comes together with a larger low-pressure area

(2) For the scenario of anisotropic permeability reservoir, a larger permeability orthogonal to the initial fracture can result in a larger cumulative production but lower increase ratio of the refracturing treatment

(3) A refracture that is orthogonal to the initial fracture can most significantly increase the low-pressure area of the refracture system, thus leading to the best performance of the refracturing treatment among different refracture azimuths

(4) A longer refracture length is more favorable for improving the well productivity. However, the length of the reoriented section can exert a more noticeable effect on the well performance than the length of the initially oriented section

(5) The refracturing treatment can lead to a higher increase of the cumulative production for the scenario of low initial fracture permeability. A higher permeability of the refracture can induce higher cumulative production

\section{Abbreviations}

A: Connection area between fracture segment and matrix grid, $\mathrm{m}^{2}$

$B$ : Formation volume factor

$d_{m-f}$ : Average distance between the fracture segment and the matrix grid, $m$

$d_{n}$ : $\quad$ Distance between the volume element and the fracture segment, $\mathrm{m}$

$\mathrm{d} V: \quad$ Volume element of the matrix grid, $\mathrm{m}^{3}$

$h$ : $\quad$ Formation thickness, $\mathrm{m}$ $k$ : Permeability, $\mathrm{mD}$

$L: \quad$ Length of fracture segment, $\mathrm{m}$

$L_{f 2 i}$ : Length of the initially orientated section, $\mathrm{m}$

$L_{f 2 r}$ : Length of the reoriented section of the refracture, $m$

p: $\quad$ Pressure, $\mathrm{MPa}$

$p_{w}: \quad$ Wellbore pressure, $\mathrm{MPa}$

$q$ : $\quad$ Flux rate, $\mathrm{m}^{3} /$ day

$Q_{\text {non }}$ : Cumulative production without refracturing treatment, $\mathrm{m}^{3}$

$Q_{\mathrm{re}}$ : Cumulative production with refracturing treatment, $\mathrm{m}^{3}$

$r_{\text {eq }}: \quad$ Equivalent radius, $\mathrm{m}$

$r_{w}$ : Wellbore radius, $\mathrm{m}$

T: $\quad$ Transmissibility, $\mathrm{m}^{3} /($ day $\bullet \mathrm{MPa})$

$V: \quad$ Volume of the matrix grid, $\mathrm{m}^{3}$

$\beta$ : $\quad$ Unit conversion factor, 0.0853

$w: \quad$ Fracture width, $\mathrm{m}$

$\varepsilon$ : Increase ratio of the cumulative production

$\mu$ : Oil viscosity, $\mathrm{mPa} \bullet$ s.

\section{Data Availability}

All data, models, and code generated or used during the study appear in the submitted article.

\section{Disclosure}

The funders had no role in the design of the study; in the collection, analyses, or interpretation of data; in the writing of the manuscript, or in the decision to publish the results.

\section{Conflicts of Interest}

The authors declare no conflict of interest.

\section{Authors' Contributions}

Formal analysis was contributed by Yawen Du. Investigation was contributed by Xiaolu Cao. Funding acquisition was contributed by Bailu Teng.

\section{Acknowledgments}

This research was funded by the Fundamental Research Funds for the Central Universities: 99959/53200659042.

\section{References}

[1] M. K. Hubbert and D. G. Willis, "Mechanics of hydraulic fracturing," Transactions of the AIME, vol. 210, no. 1, pp. 153-168, 1957.

[2] A. A. Daneshy, "Hydraulic fracture propagation in layered formations," SPE Journal, vol. 18, no. 1, pp. 33-41, 1978.

[3] E. Siebrits, J. L. Elbel, R. S. Hoover et al., "Refracture reorientation enhances gas production in Barnett shale tight gas Wells," in SPE Annual Technical Conference and Exhibition, Dallas, Texas, October 2000.

[4] X. Weng and E. Siebrits, "Effect of Production-Induced Stress Field on Refracture Propagation and Pressure Response," SPE 
Hydraulic Fracturing Technology Conference, 2007, College Station, Texas, U.S.A, January 2007, 2007.

[5] L. Shan, B. Guo, D. Weng, Z. Liu, and H. Chu, "Posteriori assessment of fracture propagation in refractured vertical oil Wells by pressure transient analysis," Journal of Petroleum Science and Engineering, vol. 168, pp. 8-16, 2018.

[6] S. Yi and M. Shaerma, "A new method to calculate slurry distribution among multiple fractures during fracturing and refracturing," Journal of Petroleum Science and Engineering, vol. 170, pp. 304-314, 2018.

[7] Y. Wang and S. Salehi, "Refracture candidate selection using hybrid simulation with neural network and data analysis techniques," Journal of Petroleum Science and Engineering, vol. 123, pp. 138-146, 2014.

[8] X. Li, J. Wang, and D. Elsworth, "Stress redistribution and fracture propagation during restimulation of gas shale reservoirs," Journal of Petroleum Science and Engineering, vol. 154, pp. 150-160, 2017.

[9] J. Wang, Y. Wei, and Y. Qi, "Semi-analytical modeling of flow behavior in fractured media with fractal geometry," Transp. Porous. Med., vol. 112, no. 3, pp. 707-736, 2016.

[10] W. Luo, C. Tang, and Y. Zhou, "A new fracture-unit model and its application to aZ-fold fracture," SPE Journal, vol. 24, no. 1, pp. 319-333, 2019.

[11] Q. Liu, S. Tian, W. Yu et al., "A semi-analytical model for simulation of multiple vertical wells with well interference," Journal of Petroleum Science and Engineering, vol. 195, article 107830, 2020.

[12] Y. Xu, X. Li, Q. Liu, S. Yang, and X. Tan, “A semi-analytical solution of finite-conductivity multi-wing fractured well in naturally fractured reservoirs by boundary element method," Journal of Petroleum Science and Engineering, vol. 203, article 108584, 2021.

[13] F. Zhang and H. Emami-Meybodi, "A Semianalytical method for two-phase flowback rate-transient analysis in shale gas reservoirs," SPE Journal, vol. 25, no. 4, pp. 1599-1622, 2020.

[14] S. H. Lee, M. F. Lough, and C. L. Jensen, "Hierarchical modeling of flow in naturally fractured formations with multiple length scales," Water Resources Research, vol. 37, no. 3, pp. 443-455, 2001.

[15] A. Sangnimnuan, J. Li, and K. Wu, "Development of efficiently coupled fluid-flow/geomechanics model to predict stress evolution in unconventional reservoirs with complex-fracture geometry," SPE Journal, vol. 23, no. 3, pp. 640-660, 2018.

[16] Y. Xu and K. Sepehrnoori, "Development of an embedded discrete fracture model for field-scale reservoir simulation with complex corner-point grids," SPE Journal, vol. 24, no. 4, pp. 1552-1575, 2019.

[17] M. Yao, H. Chang, X. Li, and D. Zhang, “An integrated approach for history matching of multiscale-fracture reservoirs," SPE Journal, vol. 24, no. 4, pp. 1508-1525, 2019.

[18] D. W. Peaceman, "Interpretation of wellblock pressures in numerical reservoir simulation with nonsquare grid blocks and anisotropic permeability," SPE Journal, vol. 23, no. 3, pp. 531-543, 1990.

[19] T. Ertekin, J. H. Abou-Kassem, and G. R. King, Basic applied reservoir simulation, Textbook Series, Society of Petroleum Engineers, Richardson, Texas, USA, 2001.
[20] J. P. Spivey and L. John, "New Solutions for Pressure Transient Response for a Horizontal or a Hydraulically Fractured Well at an Arbitrary Orientation in an Anisotropic Reservoir," in SPE Annual Technical Conference and Exhibition, New Orleans, Louisiana, September 1998.

[21] D. Kong, Y. Gao, H. Sarma, Y. Li, H. Guo, and W. Zhu, "Experimental investigation of immiscible water-alternating-gas injection in ultra-high water-cut stage reservoir," Advances In Geo-Energy Research, vol. 5, no. 2, pp. 139-152, 2021.

[22] S. Al-Rbeawi, "Performance-based comparison for hydraulically fractured tight and shale-gas reservoirs with and without non-Darcy-flow effect," SPE Reservoir Evaluation \& Engineering, vol. 21, no. 4, pp. 981-1006, 2018. 